\title{
Attractor for a Reaction-Diffusion System Modeling Cancer Network
}

\author{
Xueyong Chen, Jianwei Shen, and Hongxian Zhou \\ Institute of Applied Mathematics, Xuchang University, Xuchang, Henan 461000, China \\ Correspondence should be addressed to Jianwei Shen; xcjwshen@gmail.com
}

Received 12 January 2014; Accepted 26 March 2014; Published 17 April 2014

Academic Editor: Imran Naeem

Copyright (C) 2014 Xueyong Chen et al. This is an open access article distributed under the Creative Commons Attribution License, which permits unrestricted use, distribution, and reproduction in any medium, provided the original work is properly cited.

\begin{abstract}
A reaction-diffusion cancer network regulated by microRNA is considered in this paper. We study the asymptotic behavior of solution and show the existence of global uniformly bounded solution to the system in a bounded domain $\Omega \subset R^{n}$. Some estimates and asymptotic compactness of the solutions are proved. As a result, we establish the existence of the global attractor in $L^{2}(\Omega) \times L^{2}(\Omega)$ and prove that the solution converges to stable steady states. These results can help to understand the dynamical character of cancer network and propose a new insight to study the mechanism of cancer. In the end, the numerical simulation shows that the analytical results agree with numerical simulation.
\end{abstract}

\section{Introduction}

In this paper, we discuss the asymptoti cbehavior of solutions for reaction-diffusition equations which studied by Aguda et al. [1] and Shen et al. [2]. The system describes cancer network regulated by microRNA (miRNA). MicroRNAs are an abundant class of small noncoding RNA that function to regulate the activity and stability of specific mRNA targets through posttranscriptional regulatory mechanism and play a role of repressing translation of mRNA or degrading mRNAs. Recent studies show that miRNAs play a central role in many biological (cellular) processes, including developmental timing, cell proliferation, apoptosis, metabolism, cell differentiation, somitogenesis, and tumour-genesis. In addition, there is diffusion when molecules interact (see, e.g., $[2-6])$, so we should consider the diffusion process and its dynamical behavior.

In order to understand further the miR-17-92 involved in the network with Myc and E2F, we would investigate the cancer network $[1,2]$ with diffusion term and consider the attractor system of cancer network with diffusion as follows:

$$
\begin{gathered}
\frac{\partial u}{\partial t}=D_{1} \Delta u-\delta u+f(u, v), \quad x \in \Omega, t>0, \\
\frac{\partial v}{\partial t}=D_{2} \Delta v+k u-\gamma v, \quad x \in \Omega, t>0,
\end{gathered}
$$

$$
\begin{gathered}
\frac{\partial u}{\partial n}=\frac{\partial v}{\partial n}=0, \quad x \in \partial \Omega, \\
u(x, 0)=u_{0}(x), \quad v(x, 0)=v_{0}(x), \quad x \in \bar{\Omega},
\end{gathered}
$$

and here $\Omega$ is an open bounded subset of $R^{n}$ with the boundary of class $C^{3} . f(u, v)$ are two real smooth nonlinear functions for $u, v \in[0,+\infty)$ satisfying the following conditions:

(i) there exists $\lambda>0$ such that $f(u, v) \leq \lambda_{1} u^{2}+\lambda_{2} v$ as $u \geq 0, v \geq 0$

(ii) there exists $M_{1}, M_{2}>0$ such that $\left|f_{u}^{\prime}\right|<M_{1},\left|f_{v}^{\prime}\right|<$ $M_{2}$ for all $u, v \in[0,+\infty)$.

To our knowledge, the long time behavior of solution for reaction-diffusion system has been studied by several authors (see [7-15]). But for different nonlinear reaction function, there are some different extra difficulties. We will study the existence of global attractor for the system (1) in $L^{2}(\Omega) \times$ $L^{2}(\Omega)$. The key point to our method relies on the regularity and estimates on solutions which show that the solutions are uniformly bounded in $L^{2}(\Omega) \times L^{2}(\Omega)$.

We construct a local solution of system (1) by the semigroup method and fixed-point theorem and then discuss 
its regularity by priori estimate method. We study the asymptotic behavior of solution and show the existence of global uniformly bounded solution to the system in a bounded domain $\Omega \subset R^{n}$. Some estimates and asymptotic compactness of the solutions are proved. As a result, we establish the existence of global attractor in $L^{2}(\Omega) \times L^{2}(\Omega)$ and prove that the solution converges to stable steady states. In the end, we apply these results to the cancer network model and give the numerical test. The numerical simulation shows that the analytical results agree with numerical simulation. This paper is more motivated from the mathematical point of view than from the biological one, but it will help to get more insights in the understanding of the behavior of the problem.

For readers' convenience, the following standard result on attractor is first presented here (see, e.g., $[8,9,13,14]$ ).

Proposition 1. Suppose that $X$ is a metric space and $S(t)_{t \geq 0}$ is a semigroup of continuous operators in $X$. If $S(t)_{t \geq 0}$ has a bounded absorbing set and is asymptotically compact, then $S(t)_{t \geq 0}$ possesses a global attractor which is a compact invariant set and attracts every bounded set in $X$.

Definition 2. The semigroup $S(t)$ is asymptotically compact; that is, if $u_{n}$ is bounded in $X$ and $t_{n} \rightarrow \infty$, then $S\left(t_{n}\right) u_{n}$ is precompact in $X$.

\section{Preliminary}

Some well-known inequalities and embedding results that will be used in the sequel are presented.

Lemma 3 (see [9]). If $p, q \geq 1$ and $p(n-q)<n q$, then, for $r \in(0, p)$,

$$
\|u\|_{L^{p}(\Omega)} \leq c\|u\|_{W^{1, q}}^{a} \cdot\|u\|_{L^{r}(\Omega)}^{(1-a)} \quad \forall u \in W^{1, q}(\Omega),
$$

where $a=((n / r)-(n / p)) /(1-(n / q)+(n / r)) \in(0,1)$.

Lemma 4 (see [9]). Let $1 \leq q \leq p \leq \infty$ and $f \in L^{q}(\Omega)$. Then

$$
\begin{gathered}
\left\|e^{t \Delta} f\right\|_{p} \leq(4 \pi t)^{-(n / 2)((1 / q)-(1 / p))}\|f\|_{q} \\
\left\|(-\Delta+1)^{\beta} e^{t \Delta} f\right\|_{p} \leq c t^{-\beta-(n / 2)((1 / q)-(1 / p))} e^{(1-\mu) t}\|f\|_{q} \\
\quad p \neq q,
\end{gathered}
$$

where $\beta>0, \mu>0$, and $c$ is a positive constant depending only on $p, q, \Omega .\left(e^{t \Delta} f\right)(x)=\int_{\Omega} G(x-y, t) f(y) d y$ and $G(x, t)$ is the Green function of the heat equation $(\partial u / \partial t)-\Delta u=0$, $x \in \Omega, t>0$ with the homogeneous Neumann boundary condition.
Lemma 5 (see [10]). Let $A_{p}=-\Delta$ and $D\left(A_{p}\right)=\{\varphi \in$ $\left.W_{(\Omega)}^{2, p}|\partial \varphi / \partial N|_{\partial \Omega}=0\right\} ;$ then

$$
\begin{gathered}
D\left(\left(A_{p}+1\right)^{\beta}\right) \hookrightarrow W^{1, p}(\Omega), \quad \text { if } \beta>\frac{1}{2}, \\
D\left(\left(A_{p}+1\right)^{\beta}\right) \hookrightarrow C^{\delta}(\Omega), \quad \text { if } 2 \beta-\frac{n}{p}>\delta \geq 0, \\
\left\|(A+1)^{\beta} e^{-t(A+1)} u\right\|_{L^{p}(\Omega)} \leq c t^{-\beta}\|u\|_{L^{p}(\Omega)} .
\end{gathered}
$$

Lemma 6 (see [12]). Suppose $H^{s}$ is an interpolation space of $H^{s_{0}}$ and $H^{s_{1}}$, where $0 \leq s_{0}<s_{1}<\infty, 0<\theta<1$, and $s=$ $(1-\theta) s_{0}+\theta s_{1}$, then

$$
\|\cdot\|_{H^{s}} \leq c\|\cdot\|_{H^{s_{0}}}^{1-\theta}\|\cdot\|_{H^{s_{1}}}^{\theta}
$$

and here $H^{s}(\Omega), s>0$, denotes the fractional Sobolev space in $\Omega$.

In this paper, we denote the standard Sobolev spaces by $H^{s}$ and $H^{0}=L^{2}(\Omega)$. For any $1 \leq p \leq \infty$, we denote the norm of $L^{p}(\Omega)$ by $\|\cdot\|_{L^{p}}$. In general, $\|\cdot\|_{X}$ denotes the norm of any Banach space $X$.

\section{Local Existence and Uniqueness}

The local existence of a solution to system (1) is discussed in this section.

Theorem 7. Suppose $0 \leq u_{0}(x) \in L^{2}(\Omega), 0 \leq v_{0}(x) \in L^{2}(\Omega)$; then there is a $T \leq \infty$ (depending on initial data) such that there exists a unique nonnegative solution $(u(x, t), v(x, t))$ to (1) in $[0, T]$ and

$$
u, v \in C^{0}\left([0, T] ; L^{2}(\Omega)\right) \bigcap C^{2,1}(\Omega ;(0, T])
$$

Proof. Choose $T \in(0,1)$ and $R>0$ to be fixed. In Banach space $X=C^{0}\left([0, T] ; L^{2}(\Omega)\right) \times C^{0}\left([0, T] ; L^{2}(\Omega)\right)$, we define a bounded closed set

$$
S:=\left\{(u, v) \in X \mid\|(u, v)\|_{X} \leq R\right\} .
$$

Let

$$
\begin{aligned}
\psi(u, v)(t) & =\left(\begin{array}{l}
\psi_{1}(u, v)(t) \\
\psi_{2}(u, v)(t)
\end{array}\right) \\
& =\left(\begin{array}{c}
e^{t \Delta} u_{0}-\int_{0}^{t} e^{(t-s) \Delta}\left[-\delta_{1}+f(u, v)\right] d s \\
e^{t(\Delta-1)} v_{0}+\int_{0}^{t} e^{(t-s)(\Delta-1)} u(s) d s
\end{array}\right) .
\end{aligned}
$$


Next, we prove $\psi$ is a contractive mapping from $S$ into itself for $T$ small enough and $R$ sufficiently large. By Lemma 4 , then

$$
\begin{aligned}
\left\|\psi_{1}(u, v)\right\|_{L^{2}} \leq & \left\|e^{-t A} u_{0}(x)\right\|_{L^{2}}+\delta_{1} \int_{0}^{t}\left\|e^{-(t-s) A} u\right\|_{L^{2}} d s \\
& +\int_{0}^{t}\left\|e^{-(t-s) A} f(u(s), v(s))\right\|_{L^{2}} d s \\
\leq & \left\|u_{0}(x)\right\|_{L^{2}}+\delta_{1} \int_{0}^{t}\|u\|_{L^{2}} d s \\
& +\int_{0}^{t}(t-s)^{-(n / 2)(1-(1 / 2))}\|f(u(s), v(s))\|_{L^{1}} d s
\end{aligned}
$$

where $A=-\Delta$. For $n \leq 3,1-(n / 4)>0$, and $\int_{0}^{t}(t-$ $s)^{-(n / 2)(1-(1 / 2))}\|f(u(s), v(s))\|_{L^{1}} d s \leq c R T^{1-(n / 4)}$. Hence

$$
\left\|\psi_{1}(u, v)\right\|_{L^{2}} \leq\left\|u_{0}(x)\right\|_{L^{2}}+\delta_{1} R T+c R T^{1-(n / 4)} .
$$

By Lemma 5 , for any $t \in[0, T)$,

$$
\begin{aligned}
\left\|\psi_{2}(u, v)\right\|_{L^{2}} & \leq\left\|e^{-t(A+1)} v_{0}(x)\right\|_{L^{2}}+\int_{0}^{t}\left\|e^{-(t-s)(A+1)} u(s)\right\|_{L^{2}} d s \\
& \leq\left\|v_{0}(x)\right\|_{L^{2}}+c \int_{0}^{t} e^{-(t-s)}\|u(s)\|_{L^{2}} d s \\
& \leq\left\|v_{0}(x)\right\|_{L^{2}}+c R \int_{0}^{t} e^{-(t-s)} d s \\
& \leq\left\|v_{0}(x)\right\|_{L^{2}}+c R\left(1-e^{-T}\right) .
\end{aligned}
$$

Equations (10) and (11) imply that $\psi S \subset S$ for any fixed positive $R$ large enough and $T$ small enough. Now we show that $\psi$ is a contractive operator from $S$ to $S$. For any $(u, v),(\bar{u}, \bar{v}) \in S$, and for all $t \in[0, T)$,

$$
\begin{aligned}
\left\|\psi_{1}(u, v)-\psi_{1}(\bar{u}, \bar{v})\right\|_{L^{2}} \\
\leq c \delta_{1} \int_{0}^{t}\left\|e^{-(t-s) A}(u-\bar{u})\right\|_{L^{2}} d s \\
\quad+\int_{0}^{t}\left\|e^{-(t-s) A}(f(u, v)-f(\bar{u}, \bar{v}))\right\|_{L^{2}} d s \\
\leq c \delta_{1} T\|(u, v)-(\bar{u}, \bar{v})\|_{X} \\
\quad+M \int_{0}^{t}\|(u, v)-(\bar{u}, \bar{v})\|_{X} d s \\
\leq\left(c \delta_{1} T+M T\right)\|(u, v)-(\bar{u}, \bar{v})\|_{X}, \\
\left\|\psi_{2}(u, v)-\psi_{2}(\bar{u}, \bar{v})\right\|_{L^{2}} \\
\leq c \int_{0}^{t}\left\|e^{-(t-s)(A+1)}(u(s)-\bar{u}(s))\right\|_{L^{2}} d s \\
\leq c \int_{0}^{t} e^{-(t-s)}\|u(s)-\bar{u}(s)\|_{L^{2}} d s \\
\leq c\left(1-e^{-T}\right)\|(u, w)-(\bar{u}, \bar{w})\|_{X} .
\end{aligned}
$$

Equation (12) implies that $\psi$ is a contractive mapping if $T$ is sufficiently small. By Banach's fixed point theorem, there exists a unique fixed point $(u, v) \in X$ which is just a local solution to (1) in $X$.

Note that, for any given smooth function $v, u=0$ is the subsolution of the following problem:

$$
\begin{gathered}
\frac{\partial u}{\partial t}=\Delta u-\delta u+f(u, v), \quad x \in \Omega, t>0, \\
\frac{\partial u}{\partial n}=0, \quad x \in \partial \Omega, \\
u(x, 0)=u_{0}(x), \quad x \in \bar{\Omega},
\end{gathered}
$$

and for any given smooth function $u, v=0$ is the subsolution of the following problem:

$$
\begin{gathered}
\frac{\partial v}{\partial t}-\Delta v+v=u, \quad x \in \Omega, t>0, \\
\frac{\partial v}{\partial n}=0, \quad x \in \partial \Omega, \\
v(x, 0)=v_{0}(x), \quad x \in \bar{\Omega} .
\end{gathered}
$$

By the comparison principle, for any $t \in\left[0, T_{\max }\right)$ and $x \in \bar{\Omega}$, $u(x, t) \geq 0 ; w(x, t) \geq 0$.

Now, we discuss the regularity of the solution to (1). From the above analysis, $u(t, x), v(x, t)$ is bounded in $L^{2}(\Omega)$ for any $t \in[0, T]$. From the semigroup representation of the solution to (1) and Lemmas 3 and 4 , for any $q \in(2, n /(n-2)]$ and $\tau \in(0, T)$, there exists $\alpha=1-(n / 2)(1-(1 / q))>0$ such that

$$
\begin{aligned}
\|u(x, t)\|_{L^{q}} \leq & \left\|e^{-(t-(\tau / 2)) A} u_{(\tau / 2)}(x)\right\|_{L^{q}} \\
& +c \int_{(\tau / 2)}^{t}\left\|e^{-(t-s) A} f(u, v)\right\|_{L^{q}} d s \\
& +\delta \int_{(\tau / 2)}^{t}\left\|e^{-(t-s) A} u(s)\right\|_{L^{q}} d s \\
\leq & c\left(t-\frac{\tau}{2}\right)^{-(n / 2)((1 / 2)-(1 / q))}\left\|u_{(\tau / 2)}(x)\right\|_{L^{2}} \\
& +c \lambda \int_{(\tau / 2)}^{t}(t-s)^{-(n / 2)(1-(1 / q))}\left\|u^{2}\right\|_{L^{1}} d s \\
& +\delta \int_{0}^{t}(t-s)^{-(n / 2)((1 / 2)-(1 / q))}\|u(s)\|_{L^{2}} d s \\
\leq & c \tau^{-(n / 2)((1 / 2)-(1 / q))}\left\|u_{\tau / 2}(x)\right\|_{L^{2}} \\
& +\left(t-\frac{\tau}{2}\right)^{\alpha}\left[\frac{c \lambda}{\alpha}+\frac{\delta}{\alpha+(n / 2)}\left(t-\frac{\tau}{2}\right)^{n / 2}\right] \\
& \times \sup _{\tau / 2<s<t}\|u\|_{L^{2}},
\end{aligned}
$$

which implies that $\|u(x, t)\|_{L^{q}}$ is bounded for any $t \in[\tau, T]$. 
If $n=3$, repeating the above process, we choose $3 /(2+$ $3 \epsilon)<q_{0}<3 / 2$ for any small enough $\epsilon>0$ and $q \in[2,1 / \epsilon)$, then there exists $\alpha_{1}=1-(n / 2)\left(\left(1 / q_{0}\right)-(1 / q)\right)$ such that

$$
\begin{aligned}
& \int_{\tau / 2}^{t}\left\|e^{-(t-s) A} f(u, v)\right\|_{L^{q}} d s \\
& \quad \leq c \lambda \int_{\tau / 2}^{t}(t-s)^{-(n / 2)\left(\left(1 / q_{0}\right)-(1 / q)\right)}\left\|u^{2}\right\|_{L^{q_{0}}} d s \\
& \quad \leq c \lambda \int_{\tau / 2}^{t}(t-s)^{-(n / 2)\left(\left(1 / q_{0}\right)-(1 / q)\right)}\|u\|_{L^{2 q_{0}}} d s \\
& \quad \leq \frac{c \lambda}{\alpha_{1}}(t-s)^{\alpha_{1}} \sup _{\tau / 2<s<t}\|u\|_{L^{2 q_{0}}} .
\end{aligned}
$$

From the above analysis, for any $p \geq 2,\|u(x, t)\|_{L^{q}}(n \leq 3)$ is uniformly bounded for any $t \in[\tau, T]$. On the other hand, since $v(x, t)=e^{(t-(\tau / 2))(\Delta-1)} v_{\tau / 2}+\int_{\tau / 2}^{t} e^{(t-s)(\Delta-1)} u(s) d s$, for any $\tau \in(0, T]$, we deduce that

$$
\begin{aligned}
\|v\|_{L^{p}} \leq & \left\|e^{-(t-(\tau / 2))(A+1)} v_{\tau / 2}(x)\right\|_{L^{p}} \\
& +\int_{\tau / 2}^{t}\left\|e^{-(t-s)(A+1)} u(s)\right\|_{L^{p}} d s \\
\leq & \left(t-\frac{\tau}{2}\right)^{-(n / 2)((1 / 2)-(1 / p))}\left\|v_{\tau / 2}(x)\right\|_{L^{2}} \\
& +c \int_{0}^{\infty} e^{-(t-s)}(t-s)^{-(n / 2)((1 / q)-(1 / p))}\|u(s)\|_{L^{q}} d s \\
\leq & c_{1}(\tau)^{-(n / 2)((1 / 2)-(1 / p))}+c \Gamma(\gamma) \sup _{\tau<s<t}\|u(s)\|_{q^{\prime}}
\end{aligned}
$$

where $2 \leq q \leq p<\infty, \gamma=1-\beta-(n / 2)((1 / q)-(1 / p))$, and $\Gamma(\cdot)$ is the Gamma function. It can be proved that

$$
u, v \in C^{0}\left((0, T] ; L^{\infty}(\Omega)\right) .
$$

There exist $p, \beta, \varepsilon_{1}$ satisfying $p>n, 1 / 2>\beta>n / 2 p$ such that, for any small enough constant $\eta>0$ and $t \in[\tau+\eta, T)$,

$$
\begin{aligned}
\|u(x, t)\|_{C^{0}} \leq & \left\|e^{-(t-\tau) A} u_{\tau}(x)\right\|_{C^{0}}+c \delta \int_{\tau}^{t}\left\|e^{-(t-s) A} u\right\|_{C^{0}} d s \\
& +\int_{\tau}^{t}\left\|e^{-(t-s) A} f(u(s), v(s))\right\|_{C^{0}} d s \\
\leq & \left\|(A+1)^{\beta} e^{-(t-\tau) A} u_{\tau}(x)\right\|_{L^{p}} \\
& +c \delta \int_{\tau}^{t}\left\|(A+1)^{\beta} e^{-(t-s) A} u(s)\right\|_{L^{p}} d s \\
& +\int_{\tau}^{t}\left\|(A+1)^{\beta} e^{-(t-s) A} f(u(s), v(s))\right\|_{L^{p}} d s \\
\leq & \eta^{-\beta-(n / 2)((1 / 2)-(1 / p))} e^{(1-\mu) t}\left\|u_{\tau}(x)\right\|_{L^{2}} \\
& +c \delta \sup _{\tau<s<t}\|u\|_{L^{2}} \int_{\tau}^{t}(t-s)^{-\beta-(n / 2)((1 / 2)-(1 / p))} d s \\
& +c \lambda \sup _{\tau<s<t}\|u\|_{L^{4}} \int_{\tau}^{t}(t-s)^{-\beta-\varepsilon_{1}} d s,
\end{aligned}
$$

where $\varepsilon_{1}=(n / 2)((1 / 2)-(1 / p))<1-\beta$, which implies that $u \in C^{0}\left((0, T] ; C^{0}(\Omega)\right)$. By semigroup techniques and Schauder estimates, we see that

$$
u(x, t), v(x, t) \in C\left([0, T] ; L^{2}(\Omega)\right) \bigcap C^{2,1}(\Omega ;(0, T]) .
$$

It easy to know that

$$
u, v \in C^{0}\left([0, T] ; L^{2}(\Omega)\right) \bigcap C^{0}\left((0, T] ; H_{N}^{2}(\Omega)\right),
$$

and here we denote that $H_{N}^{s}=\left\{u \in H^{s}(\Omega) ; \partial u / \partial n=\right.$ 0 , on $\partial \Omega\}$.

\section{Global Solution and Some A Priori Estimates}

In this section, the global-in-time existence of a solution to system (1) is proved. The following a priori estimates will play a crucial role in the proof of our result.

Lemma 8. Suppose that $0 \leq u_{0} \in L^{2}(\Omega), 0 \leq v_{0} \in L^{2}(\Omega)$, and $(u, v)$ is a local solution to $(1)$ in $[0, T]$ satisfying

$$
\begin{array}{r}
u, v \in C^{0}\left([0, T] ; L^{2}(\Omega)\right) \bigcap C^{0}\left((0, T] ; H^{1}(\Omega)\right) \\
\bigcap C^{0}\left((0, T] ; H_{N}^{2}(\Omega)\right),
\end{array}
$$

then, for any $\tau>0$,

$$
\begin{array}{r}
\|u(t)\|_{L^{2}}^{2}+\|v(t)\|_{L^{2}}^{2} \leq e^{-v_{1} t}\left(c t+\left(\left\|u_{0}\right\|_{L^{2}}^{2}+\left\|v_{0}\right\|_{L^{2}}^{2}\right)\right), \\
0 \leq t \leq T, \\
\|u(t)\|_{H^{1}}+\|v(t)\|_{H^{1}} \leq C, \quad \tau<t \leq T,
\end{array}
$$

where $C$ depends only on $\Omega, u_{\tau}, v_{\tau}$, and $v_{1}>0$.

Proof. In the process of the proof, we denote any positive constant by $c$ which may change from line to line and let $\tau$ be a small enough constant.

Step 1. Taking the inner product of the first equation of (1) with $u$ in $L^{2}(\Omega)$, by Young's inequality, for any $\varepsilon>0$, there is a constant $c_{\varepsilon}$ such that

$$
\begin{gathered}
\frac{1}{2} \frac{d\|u(t)\|_{L^{2}}^{2}}{d t}+\int_{\Omega}|\nabla u|^{2} d x+\delta \int_{\Omega} u^{2} d x \\
\quad=\int_{\Omega} f(u, v) u d x \\
\quad \leq \varepsilon \int_{\Omega}|u|^{2} d x+c_{\varepsilon}|\Omega| .
\end{gathered}
$$


Taking the inner product of the second equation of (1) with $v$ in $L^{2}(\Omega)$, for any $\varepsilon_{1}>0$,

$$
\begin{aligned}
& \frac{1}{2} \frac{d\|v(t)\|_{L^{2}}^{2}}{d t}+\int_{\Omega}|\nabla v|^{2} d x+\gamma \int_{\Omega}|v|^{2} d x \\
& \quad=k_{2} \int_{\Omega} u v d x \\
& \quad \leq \frac{\varepsilon_{1}^{2} k_{2}}{2} \int_{\Omega}|u|^{2} d x+\frac{1}{2 k_{2} \varepsilon_{1}^{2}} \int_{\Omega}|v|^{2} d x .
\end{aligned}
$$

If these parameters meet the conditions, $4 \gamma \delta \geq k_{2}$, then there are $\varepsilon, \varepsilon_{1}$ such that $v_{1}=\min \left\{\delta-\varepsilon-\left(\varepsilon_{1}^{2} / 2\right), 2 \gamma-\left(1 / \varepsilon_{1}^{2}\right)\right\}>0$. From (24) and (25),

$$
\begin{aligned}
& \frac{d\left(\|v(t)\|_{L^{2}}^{2}+\|u(t)\|_{L^{2}}^{2}\right)}{d t} \\
& \quad+v_{1}\left(\int_{\Omega}|v|^{2} d x+\int_{\Omega}|\nabla v|^{2} d x\right. \\
& \left.\quad+\int_{\Omega}|u|^{2} d x+\int_{\Omega}|\nabla u|^{2} d x\right) \leq c .
\end{aligned}
$$

By the Poincaré inequality,

$$
\int_{\Omega}|\nabla u|^{2} d x \geq c \int_{\Omega} u^{2} d x, \quad \int_{\Omega}|\nabla v|^{2} d x \geq c \int_{\Omega}|v|^{2} d x .
$$

Gronwall's lemma implies that

$$
\begin{array}{r}
\|u(t)\|_{L^{2}}^{2}+\|v(t)\|_{L^{2}}^{2} \leq c+e^{-v_{1} t}\left(\left\|u_{0}\right\|_{L^{2}}^{2}+\left\|v_{0}\right\|_{L^{2}}^{2}\right), \\
t \in[0, T] .
\end{array}
$$

Step 2. From the analysis in Step $1,\|u(t)\|_{L^{2}},\|v(t)\|_{L^{2}}$ is uniformly bounded in $[0, T]$.

Taking the inner product on both sides of the second equation with $-\Delta v$, for any $\varepsilon_{2}>0$,

$$
\begin{aligned}
& \frac{1}{2} \frac{d\|\nabla v\|_{L^{2}}^{2}}{d t}+\int_{\Omega}|\Delta v|^{2} d x+\int_{\Omega}|\nabla v|^{2} d x \\
& \quad=\int_{\Omega} \nabla u \cdot \nabla v d x \\
& \quad \leq \frac{1}{2} \int_{\Omega}|\nabla v|^{2} d x+\frac{1}{2} \int_{\Omega}|\nabla u|^{2} d x .
\end{aligned}
$$

Taking the inner product of the first equation of (1) with $-\Delta u$ in $L^{2}(\Omega)$, then

$$
\begin{aligned}
& \frac{1}{2} \frac{d\|\nabla u\|_{L^{2}}^{2}}{d t}+\int_{\Omega}|\Delta u|^{2} d x+\int_{\Omega}|\nabla u|^{2} d x \\
& \quad=\int_{\Omega}\left(f_{u} \nabla u \cdot \nabla u+f_{v} \nabla v \cdot \nabla u\right) d x \\
& \quad \leq M_{1} \int_{\Omega}|\nabla u|^{2} d x+M_{2} \int_{\Omega}|\nabla v \cdot \nabla u| d x \\
& \quad \leq\left(M_{1}+\frac{M_{2}}{2}\right) \int_{\Omega}|\nabla u|^{2} d x+\frac{M_{2}}{2} \int_{\Omega}|\nabla v|^{2} d x .
\end{aligned}
$$

By Lemma 6, there exist $\theta=1 / 2, s_{0}=0$, and $s_{1}=2$ such that

$$
\begin{aligned}
\int_{\Omega}|\nabla u|^{2} d x & \leq c\|u\|_{H^{1}}^{2} \\
& \leq c\|u\|_{H^{0}}^{2(1-\theta)}\|u\|_{H^{2}}^{2 \theta} \\
& \leq c\|u\|_{L^{2}}\|u\|_{H^{2}} .
\end{aligned}
$$

Since $\|u\|_{L^{2}}$ is bounded, for any $\varepsilon_{2}>0$, there is a constant $c_{\varepsilon_{2}}$ such that

$$
c\|u\|_{L^{2}}\|u\|_{H^{2}} \leq \varepsilon_{2}\|\Delta u\|_{L^{2}}^{2}+c_{\varepsilon_{2}} .
$$

With the same analysis, it easy to know that there exist $\varepsilon_{3}>0$, $c_{\varepsilon_{3}}$ such that

$$
\int_{\Omega}|\nabla u|^{2} d x \leq \varepsilon_{3}\|\Delta v\|_{L^{2}}^{2}+c_{\varepsilon_{3}} .
$$

From the above analysis and (29) and (30), we choose $\varepsilon_{2}, \varepsilon_{3}$ small enough; then there exists positive constant $v_{2}=$ $1 / 2>0, c_{\varepsilon_{2}, \varepsilon_{3}}>0$ such that

$$
\begin{aligned}
& \frac{1}{2} \frac{d\left(\|\nabla v\|_{L^{2}}^{2}+\|\nabla u\|_{L^{2}}^{2}\right)}{d t}+v_{2} \int_{\Omega}\left(|\nabla v|^{2}+|\nabla u|^{2}\right) d x \\
& \quad \leq c_{\varepsilon_{2}}\left(M_{1}+\frac{M_{2}}{2}\right)+\frac{M_{2} c_{\varepsilon_{3}}}{2} \leq c_{\varepsilon_{2}, \varepsilon_{3}} .
\end{aligned}
$$

By Gronwall's lemma,

$$
\begin{array}{r}
\|v\|_{H^{1}}+\|u\|_{H^{1}} \leq c_{\varepsilon_{2}, \varepsilon_{3}}+e^{-v_{2} t}\left(\left\|v_{\tau}\right\|_{H^{1}}+\left\|u_{\tau}\right\|_{H^{1}}\right) \\
t \in(\tau, T] .
\end{array}
$$

In this section, we denote any positive constant by $c$ whose value may change from line to line. Equations (30), (35) and the choice of $T$ (in Theorem 7) depending on $\left\|u_{0}\right\|_{L^{2}(\Omega)}+$ $\left\|v_{0}\right\|_{L^{2}(\Omega)}$, then $(u(x, T), w(x, T)) \in S$; it is clear by a standard argument that the solution $(u, w)$ to (1) can be extended up to some $T_{\max } \leq \infty$. With the same method as in the proof of Lemma 8 , for any finite $T_{\max }$,

$$
\begin{gathered}
\left\|u\left(T_{\max }\right)\right\|_{L^{2}}^{2}+\left\|v\left(T_{\max }\right)\right\|_{L^{2}}^{2} \leq c+e^{-v T_{\max }}\left(\left\|u_{0}\right\|_{L^{2}}^{2}+\left\|v_{0}\right\|_{L^{2}}^{2}\right), \\
\left\|u\left(T_{\max }\right)\right\|_{H^{1}}+\left\|v\left(T_{\max }\right)\right\|_{H^{1}} \leq C,
\end{gathered}
$$

which implies that $T_{\max }=+\infty$. The global existence of the solution to (1) is obtained as the following theorem.

Theorem 9. Suppose that nonnegative functions $u_{0} \in L^{2}(\Omega)$, $v_{0} \in L^{2}(\Omega)$; then there is a unique nonnegative global solution $(u, v)$ to $(1)$ satisfying

$$
\begin{array}{r}
u, v \in C\left([0, \infty) ; L^{2}(\Omega)\right) \bigcap C^{1}\left((0, \infty) ; L^{2}(\Omega)\right) \\
\bigcap C\left((0, \infty) ; H^{1}(\Omega)\right), \\
\|u(t)\|_{L^{2}}^{2}+\|v(t)\|_{L^{2}}^{2} \leq e^{-v t}\left(c t+\left(\left\|u_{0}\right\|_{L^{2}}^{2}+\left\|v_{0}\right\|_{L^{2}}^{2}\right)\right), \\
t \geq 0, \\
\|u(t)\|_{H^{1}}+\|v(t)\|_{H^{1}} \leq C, \quad t>T_{1}+1,
\end{array}
$$


where $C$ depends only on $\Omega, u_{T_{1}}, v_{T_{1}}$, and $T_{1}=(1 /$ v) $\ln \left(\left\|u_{0}\right\|_{L^{2}}^{2}+\left\|w_{0}\right\|_{L^{2}}^{2}\right)$.

\section{Global Attractor}

The existence of a global attractor to system (1) is given in this section.

From the estimates in Lemma 8, there exists fixed constant $M>0$ and $T_{1}=(1 / v) \ln \left(\left\|u_{0}\right\|_{L^{2}}^{2}+\left\|v_{0}\right\|_{L^{2}}^{2}\right)$ such that

$$
\|u(t)\|_{L^{2}}+\|v(t)\|_{L^{2}} \leq M, \quad t>T_{1} .
$$

Denote the set $B=\left\{(u, v) \in L^{2}(\Omega) \times L^{2}(\Omega):\|(u, v)\|_{L^{2} \times L^{2}} \leq\right.$ $M ; u \geq 0, v \geq 0\}$ where $M$ is the constant in (38). The results of Theorem 9 imply that the existence of a dynamical system $\{S(t)\}_{t \geq 0}$ which maps $V=\left\{(u, v):(u, v) \in L^{2} \times L^{2} ; u \geq 0, v \geq\right.$ $0\}$ into itself and satisfying $(u(t), v(t))=S(t)\left(u_{0}, v_{0}\right)$. Since $B$ is bounded, by Lemma 8 , there exists $T(B)$ depending only on $B$ and $|\Omega|$ such that

$$
S(t) B \subset B, \quad t \geq T(B),
$$

which implies that $B$ is a bounded absorbing set of the semigroup $\{S(t)\}_{t \geq 0}$.

Next, by the Sobolev embedding theorem, the asymptotical compactness of the semigroup $\{S(t)\}_{t \geq 0}$ is shown and then the existence of a global attractor to system (1) is given.

Theorem 10. Assume that $f(u, v)$ satisfying hypothesis. Then the problem (1) has a global attractor which is a compact invariant set and attracts every bounded set in $V$.

Proof. If $\left(u_{n}, v_{n}\right)$ is bounded in $V$, assume that there exists $R$ such that $\left\|\left(u_{n}, v_{n}\right)\right\|_{V} \leq R$. Then by Lemma 8 , there is a constant $T_{1}(R)$ (depending on $R$ ) such that

$$
\left(u^{n}(t), v^{n}(t)\right)=S(t)\left(u_{n}, v_{n}\right) \subset B, \quad t \geq T_{1}(R),
$$

where $B$ is the absorbing set given in (39) and $n=1,2, \ldots$ For any sequence $t_{n}\left(t_{n} \rightarrow+\infty\right.$ as $\left.n \rightarrow \infty\right)$, there is $N(R)$ such that, for any $n>N(R), t_{n} \geq T_{1}(R)+1$, and

$$
\begin{aligned}
& \left(u^{n}\left(t_{n}\right), v^{n}\left(t_{n}\right)\right) \\
& \quad=S\left(t_{n}-T_{1}(R)-1\right)\left(S\left(T_{1}(R)+1\right)\left(u_{n}, v_{n}\right)\right) .
\end{aligned}
$$

Since the two embedding $H^{1}(\Omega) \hookrightarrow L^{2}(\Omega)$ are compact, from the estimates in Theorem 9 , it is clear that $\left\{u^{n}\left(t_{n}\right)\right\}$ lies in a compact set in $L^{2}(\Omega)$ and $\left\{v^{n}\left(t_{n}\right)\right\}$ lies in a compact set in $L^{2}(\Omega)$. Hence, $\left\{\left.S\left(t_{n}\right)\left(u_{n}, v_{n}\right)\right|_{\Omega}: n=1,2 \ldots\right\}$ is precompact in $L^{2}(\Omega) \times L^{2}(\Omega)$, which implies that $\{S(t)\}_{t \geq 0}$ is asymptotically compact. We have obtained the bounded absorbing set of $\{S(t)\}_{t \geq 0}$. Then by Proposition 1, we obtain the existence of the global attractor to (1).

From the estimates in Theorem 9, it is easy to know that the solution of system (1) is exponential decay in space $L^{2}(\Omega) \times L^{2}(\Omega)$ if the forcing term $f$ is zero. So the global attractor reduces to the single point $(0,0)$.

\section{Conclusions and Discussion}

In this paper, by the semigroup method and fixed-point theorem, we construct a local solution of system (1) and then discuss its regularity by a priori estimate method. We also study the asymptotic behavior of solution and show the existence of global uniformly bounded solution to the system in a bounded domain $\Omega \subset R^{n}$. Some estimates and asymptotic compactness of the solutions are proved. To prove the compactness of the semigroup, we used the fact that Sobolev embedding is compact in bounded domains. As a result, we establish the existence of the global attractor in $L^{2}(\Omega) \times L^{2}(\Omega)$ and prove that the solution converges to stable steady states if the forcing term $f$ is zero.

The hypothesis of the nonlinear function can be more general. For the case of unbounded domains or partly dissipative system, the dynamical system (1) is not compact. Then, using the similar idea in [11], we should decompose the semigroup into two parts such that one part asymptotically tends to zero and the other part is compact. But the lack of compactness of Sobolev embedding introduces some extra difficulties. In general, the space domain should be bounded in a biological process.

The arguments in the previous sections can be applied to more general reaction diffusion systems. As we discussed in the introduction, in order to understand further the miR17-92 involving in the network with Myc and E2F, scientists plan to model this network with mathematical model. By using the mathematical model, the researchers can detect the key points regulating main properties of biological system and find the methods to solve the different diseases. In order to explain the cancer mechanism induced by miR-17-94, Aguda et al. [1] gave the ODE model and investigated different possible designs of the silencing mechanism exerted by miR17-94; the network is described by the following equations:

$$
\begin{gathered}
\frac{\partial p}{\partial t}=\alpha+\frac{k_{1} p^{2}}{a+p^{2}+b m}-\delta p \\
\frac{\partial m}{\partial t}=\beta+k_{2} p-\gamma m .
\end{gathered}
$$

Shen et al. [2] modified the system and added the diffusion to the system and obtained the reaction-diffusing system as follows:

$$
\begin{gathered}
\frac{\partial p}{\partial t}=\alpha+\frac{k_{1} p^{2}}{a+p^{2}+b m}-\delta p+D_{1} \Delta p \\
\frac{\partial m}{\partial t}=\beta+k_{2} p-\gamma m+D_{2} \Delta m
\end{gathered}
$$

where $p(x, t)$ represents the density of the protein module (Myc and the E2Fs) and $m(x, t)$ denotes the miRNA cluster. $\alpha, \beta, \delta, a, b$, and $k_{i}$ are nonnegative parameters. Let $b_{0}=$ $(1 / \gamma)\left(\beta+\left(k_{2} \alpha / \delta\right)\right)$, by the translation transformation $u=$ $p-(\alpha / \delta), v=m-b_{0}$, the cancer network system (43) is changed into a special case of system (1).

From the estimates in Theorem 9, it is easy to know that the solution of system (1) is exponential decay in 


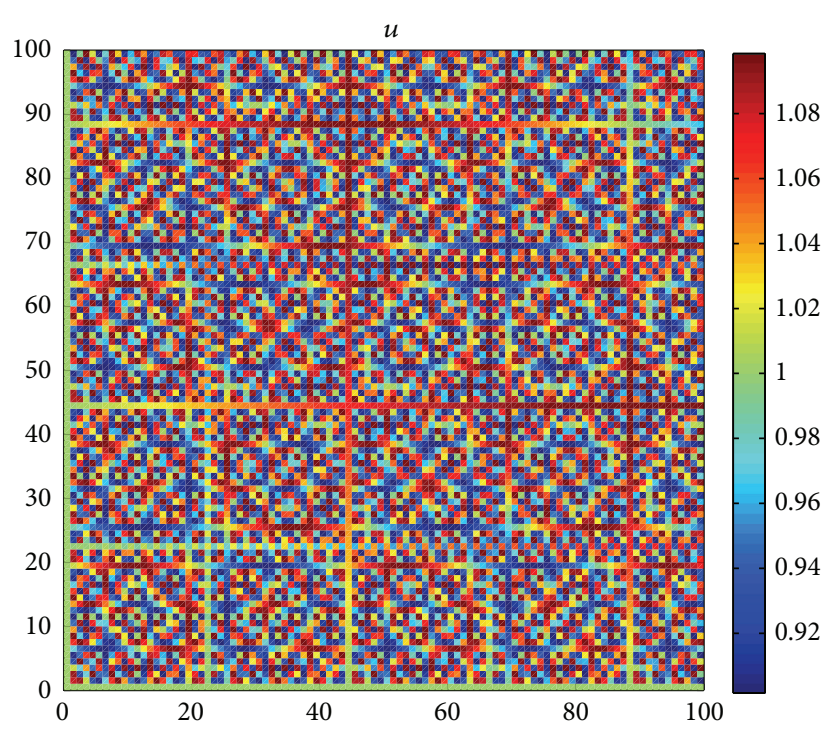

(a)

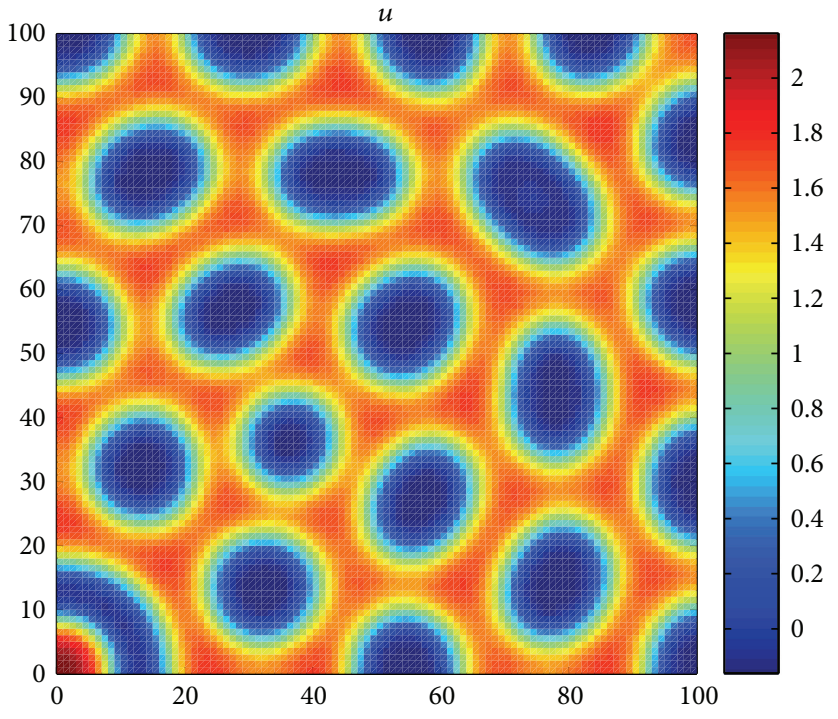

(c)

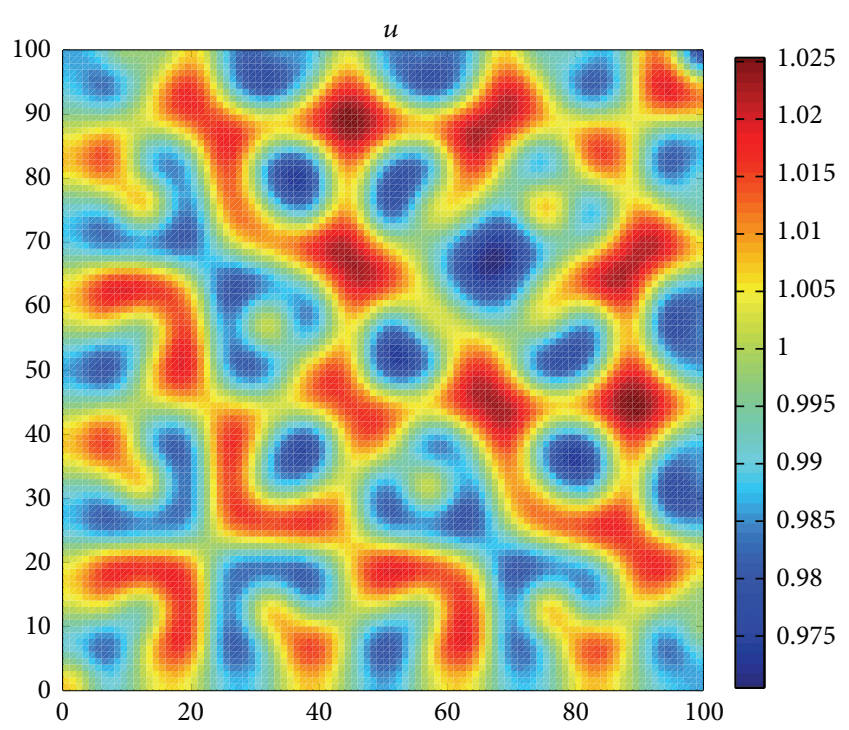

(b)

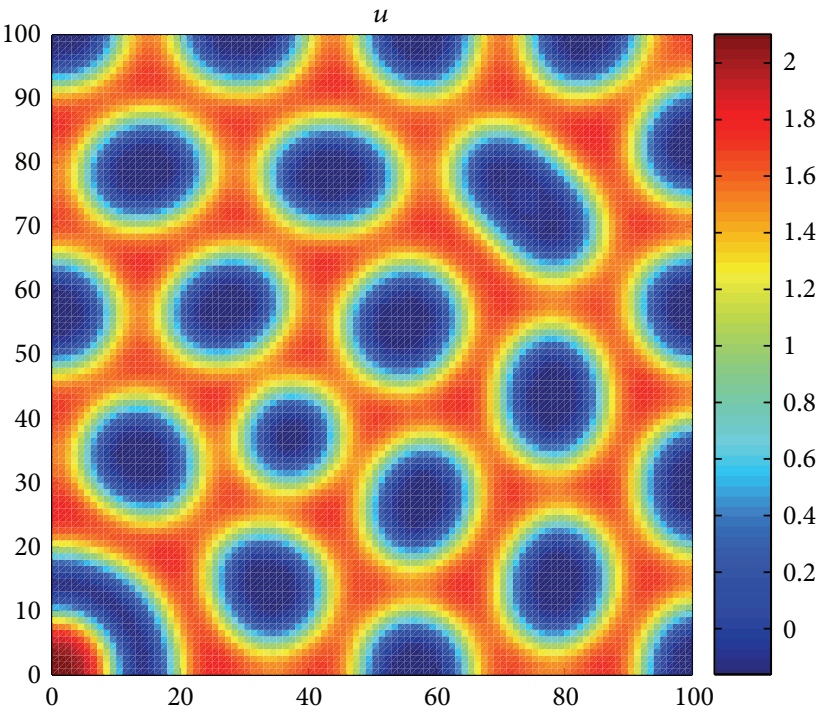

(d)

FIGURE 1: The spotted patterns prevail over the two-dimensional space. And $[(\mathrm{a})-(\mathrm{d})]$ are, respectively, at time $0,10,500,1000$. Parameter values and initial perturbation, respectively, are $\delta=0.3, \gamma=0.2,0.1 \sin (x y), 0.1 \cos (x y)$.

space $L^{2}(\Omega) \times L^{2}(\Omega)$ if the forcing term $f$ is zero. So the global attractor reduces to the single point $(0,0)$. Since the translation transformation $u=p-(\alpha / \delta), v=m-b_{0}$, which means that the solutions of the cancer network converges to stable steady states as $k_{1}=0$. From the analysis of the local solution in Section 3, the solution of the system (43) is nonnegative for any nonnegative initial value. Then, for $k_{1} \neq 0$ condition, we have $0 \leq k_{1} p^{2} /\left(a+p^{2}+b m\right) \leq k_{1}$. Let

$$
\begin{array}{ll}
\bar{p}_{0}=\sup _{x \in \Omega} p_{0}(x), & \bar{m}_{0}=\sup _{x \in \Omega} m_{0}(x), \\
\underline{p}_{0}=\inf _{x \in \Omega} p_{0}(x), & \underline{m}_{0}=\inf _{x \in \Omega} m_{0}(x) .
\end{array}
$$

Then there are a supersolution $\bar{p}(x, t)$ and a subsolution $p(x, t)$ to the first equation of the system (43) which read as follows:

$$
\begin{gathered}
\bar{p}(x, t)=\frac{\alpha+k_{1}}{\delta}+\left(\bar{p}_{0}-\frac{\alpha+k_{1}}{\delta}\right) e^{-\delta t}, \\
\underline{p}(x, t)=\frac{\alpha}{\delta}+\left(\underline{p}_{0}-\frac{\alpha}{\delta}\right) e^{-\delta t},
\end{gathered}
$$

where $\bar{p}(x, t)$ and $p(x, t)$ are the solutions of the two following equations, respectively:

$$
\begin{gathered}
\frac{d \bar{p}(x, t)}{d t}=\alpha+k_{1}-\delta \bar{p}(x, t), \quad t>0, \\
\bar{p}(x, 0)=\bar{p}_{0} \geq 0,
\end{gathered}
$$




$$
\begin{gathered}
\frac{d \underline{p}(x, t)}{d t}=\alpha-\delta \underline{p}(x, t), \quad t>0, \\
\underline{p}(x, 0)=\underline{p}_{0} \geq 0 .
\end{gathered}
$$

By comparison principle, we see the solution of the system (43) $p(x, t)$ satisfying $p(x, t) \leq p(x, t) \leq \bar{p}(x, t)$ for any $t>0$. So

$$
\lim _{t \rightarrow \infty} p(x, t)=C_{1}(x) \in\left[\frac{\alpha}{\delta}, \frac{\alpha+k_{1}}{\delta}\right],
$$

which implies that there exists a sufficiently large $T$ such that $\alpha / \delta \leq p(x, t) \leq\left(\alpha+k_{1}\right) / \delta$ for $t>T$. With the same analysis, we know that there exist a supersolution $\bar{m}(x, t)$ and a subsolution $\underline{m}(x, t)$ to the second equation of the system (43) which read as follows:

$$
\begin{aligned}
& \bar{m}(x, t)=b_{1}+\left(\bar{m}_{0}-b_{1}\right) e^{-\gamma t}, \\
& \underline{m}(x, t)=b_{0}+\left(\underline{m}_{0}-b_{0}\right) e^{-\gamma t},
\end{aligned}
$$

where $b_{0}=(1 / \gamma)\left(\beta+\left(k_{2} \alpha / \delta\right)\right)$ and $b_{1}=b_{0}+\left(k_{1} k_{2} / \delta\right)$. Then we have that

$$
\lim _{t \rightarrow \infty} m(x, t)=C_{2}(x) \in\left[b_{0}, b_{1}\right]
$$

The above analysis means that the attractor of the system (43) is a stable steady states or limit cycle.

Next, we will give the numerical test to the gene network model. The gene network model is simulated numerically in two spatial dimensions. Our numerical simulations employ the zero-flux boundary conditions. We set time step and space step as 0.02 and 1 and select coefficients of diffusion $\left(D_{1}, D_{2}\right)=(1,1)$; we choose parameters $(a, b)=(1,1)$, $\left(k_{1}, k_{2}, \alpha, \beta\right)=(1,0.1, \delta, \gamma)$.

The numerical simulation shows that the behavior of the solution to system (43) is a stable steady states or limit cycle (see Figures 1(c) and 1(d)). The numerical simulations agree with analytical results.

\section{Conflict of Interests}

The authors declare that there is no conflict of interests regarding the publication of this paper.

\section{Acknowledgments}

This work is supported by National Natural Science Foundation of China (11272277 and 11301455), Foundation of Henan Educational Committee (13A110737 and 13A110756), Program for New Century Excellent Talents in University (NCET10-0238), the Key Project of Chinese Ministry of Education (211105), Innovation Scientists and Technicians Troop Construction Projects of Henan Province (134100510013), and Innovative Research Team in University of Henan Province (13IRTSTHN019).

\section{References}

[1] B. D. Aguda, Y. Kim, M. G. Piper-Hunter, A. Friedman, and C. B. Marsh, "MicroRNA regulation of a cancer network: consequences of the feedback loops involving miR-17-92, E2F, and Myc," Proceedings of the National Academy of Sciences of the United States of America, vol. 105, no. 50, pp. 19678-19683, 2008.

[2] J. Shen, L. Chen, and K. Aihara, "Self-induced stochastic resonance in a cancer network of microRNA regulation," in Lecture Notes in Operations Research, vol. 13, pp. 251-257, 2010.

[3] V. Ambros, “The functions of animal microRNAs," Nature, vol. 431, no. 7006, pp. 350-355, 2004.

[4] J. D. Murray, Mathematical Biology. I: An Introduction, vol. 17 of Interdisciplinary Applied Mathematics, Springer, 3rd edition, 2002.

[5] S. Kondo and T. Miura, "Reaction-diffusion model as a framework for understanding biological pattern formation," Science, vol. 329, no. 5999, pp. 1616-1620, 2010.

[6] A. L. Gartel and E. S. Kandel, "miRNAs: little known mediators of oncogenesis," Seminars in Cancer Biology, vol. 18, no. 2, pp. 103-110, 2008.

[7] X. Chen and W. Liu, "Global attractor for a density-dependent sensitivity chemotaxis model," Acta Mathematica Scientia B, vol. 32, no. 4, pp. 1365-1375, 2012.

[8] R. Temam, Infinite-Dimensional Dynamical Systems in Mechanics and Physics, vol. 68, Springer, New York, NY, USA, 2nd edition, 1997.

[9] A. Friedman, Partial Differential Equations, Holt, Rinehart and Winston, New York, NY, USA, 1969.

[10] A. Pazy, Semigroups of Linear Operators and Applications to Partial Differential Equations, vol. 44, Springer, New York, NY, USA, 1983.

[11] A. Rodriguez-Bernal and B. Wang, "Attractors for partly dissipative reaction diffusion systems in $R^{n}$," Journal of Mathematical Analysis and Applications, vol. 252, no. 2, pp. 790-803, 2000.

[12] K. Osaki, T. Tsujikawa, A. Yagi, and M. Mimura, "Exponential attractor for a chemotaxis-growth system of equations," Nonlinear Analysis: Theory, Methods \& Applications, vol. 51, pp. 119144, 2002.

[13] A. V. Babin and M. I. Vishik, "Attractors of partial differential evolution equations in an unbounded domain," Proceedings of the Royal Society of Edinburgh A: Mathematics, vol. 116, no. 3-4, pp. 221-243, 1990.

[14] X. Chen and J. Shen, "Global attractor for a chemotaxis model with reaction term," Journal of Applied Mathematics, vol. 2013, Article ID 536381, 8 pages, 2013.

[15] L. E. Payne and B. Straughan, "Decay for a Keller-Segel chemotaxis model," Studies in Applied Mathematics, vol. 123, no. 4, pp. 337-360, 2009. 


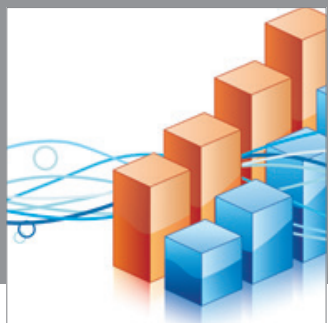

Advances in

Operations Research

mansans

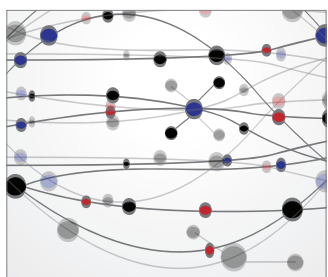

The Scientific World Journal
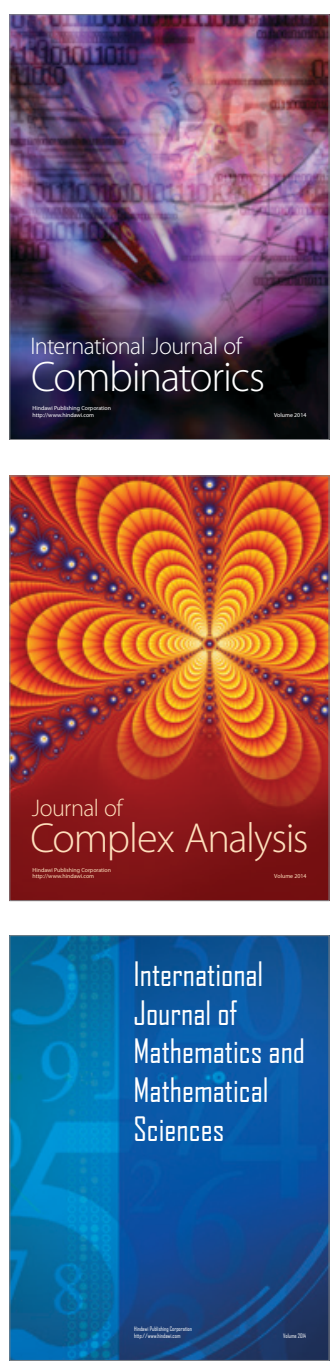
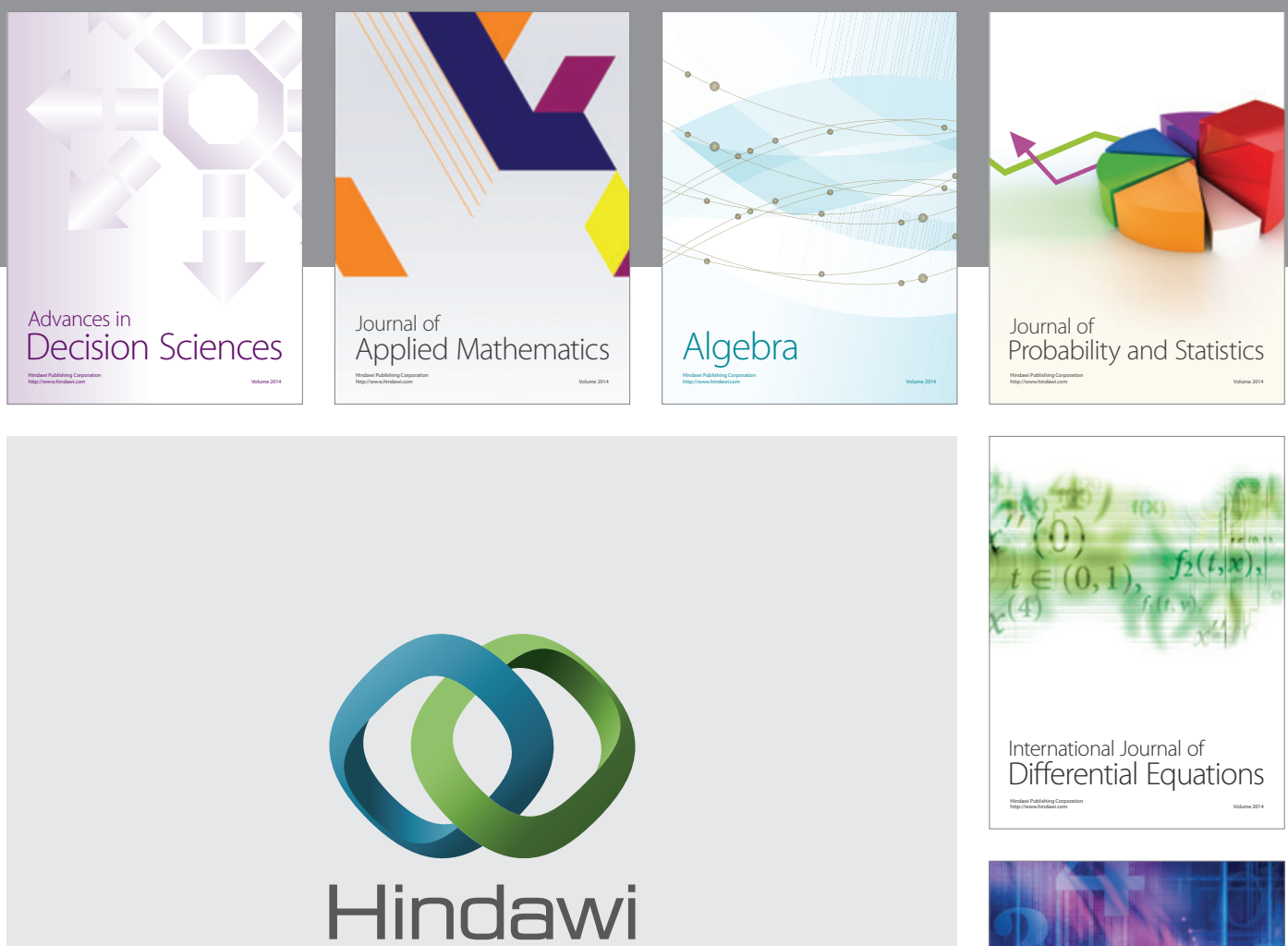

Submit your manuscripts at http://www.hindawi.com
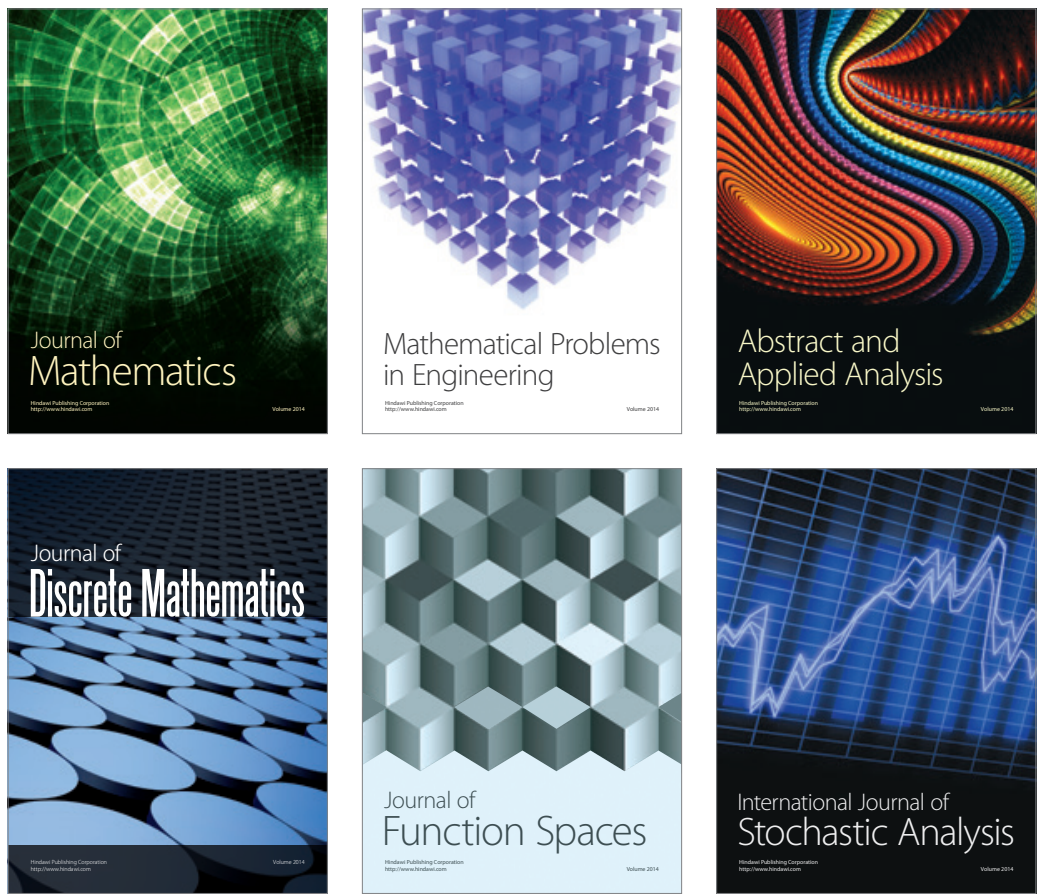

Journal of

Function Spaces

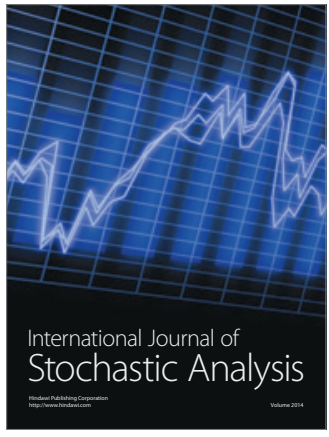

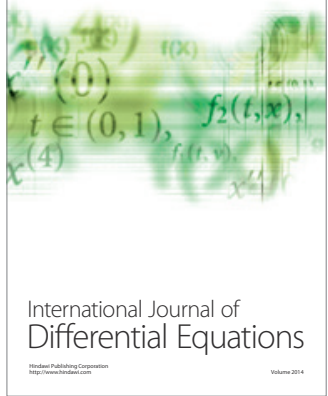
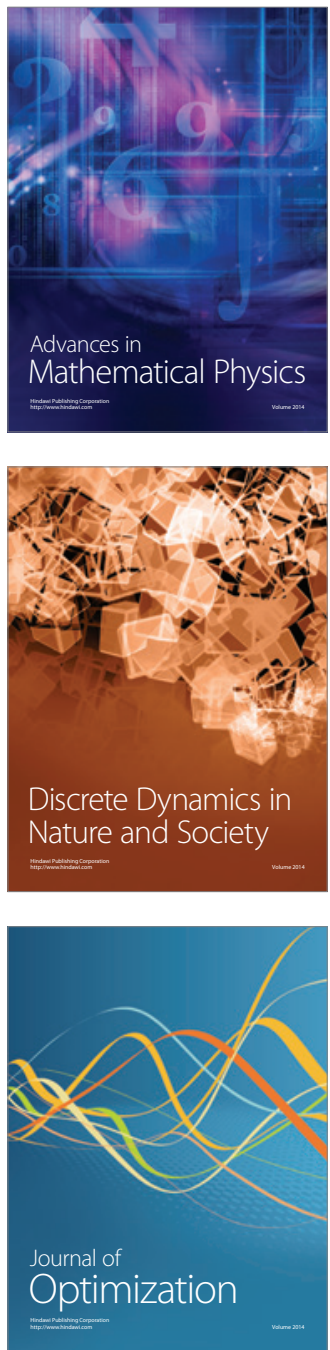